\title{
New Technique for Controlling Bleeding in Laparoscopic Liver Resection
}

\author{
Luigi Masoni ${ }^{1}$, Leandro Landi, $\mathrm{MD}^{2}$, Riccardo Maglio, $\mathrm{MD}^{3}$ \\ ${ }^{1}$ Universitary Researcher Department General Surgery, Sapienza University of Rome \\ ${ }^{2}$ University of Naples Vanvitelli \\ ${ }^{3}$ Department of General Surgery, Hospital Veris Delli Ponti Scorrano (Le)
}

*Corresponding author - Dr Riccardo Maglio; riccardomaglio@ hotmail.it

Received 24 February 2019;

Accepted 12 March 2019;

Published 15 March 2019

\begin{abstract}
Introduction: The liver is a vascular-rich solid organ. Safe and effective dissection of the vessels and liver parenchyma, and control of intraoperative bleeding are the main concerns when performing liver resection.

Controlling bleeding during laparoscopic hepatectomy $(\mathrm{LH})$ is technically demanding, but reportedly associated with less estimated blood loss (EBL) than open surgery. Several studies have confirmed that intraoperative blood loss and postoperative transfusion are predictors of postoperative morbidity and mortality in liver surgery. Various methods and instruments have been developed during hepatectomy.

Case presentation: The present study aimed to describe and evaluate hemorrhage control techniques during left lateral partial segmentectomy with use of endoscopic clips.

Conclusion: control of intraoperative bleeding it is the most important aspect in liver resection. we propose a new technique for controlling bleeding in restricted operating spaces.
\end{abstract}

\section{Introduction}

Major liver resections were initially considered unsuitable for laparoscopy since they are technically demanding and carry high risks of severe haemorrhage ${ }^{[1]}$.

The incidence of major haemorrhage varies between $0-3 \%$. Bleeding control during laparoscopic liver resection still remains an important issue, as asserted by several authors ${ }^{[2,3]}$ and it represents a challenge even for the most experienced operators, for several reasons: 1) reduction of visibility in the surgical field ${ }^{[5,6]} 2$ ) need for conversion to laparotomy ${ }^{[7]} 3$ ) lack of tactile sense may lead to a wrong resection of the lesion ${ }^{[8]}$ 4) control of major bleeding may require high pneumoperitoneum pressure setting ${ }^{[9,10]}$ with risks of gas embolism 5) a demonstrated negative effect on post-operative and oncological outcomes ${ }^{[11,12]}$. Good knowledge of all laparoscopic haemostatic devices and techniques is therefore mandatory ${ }^{[5]}$. We report on the use of endoscopic clips in a case where an important bleeding of the left suprahepatic vein did not allow safe suturing of the vessel. This case report is in line with the SCARE guidelines ${ }^{[13]}$.

\section{Case presentation}

A 47 years old Caucasian male is admitted in our Unit to undergo a left lateral partial segmentectomy (II and partial III segment resection). The patient had been previously operated by excision of left arm melanoma and a single hepatic metastasis was found in the course of a follow-up CT scan. The metastasis was located in the subdiaphragmatic part of the left liver.

The patient underwent laparoscopic resection of II-III segment. A four trocar technique was used inserting the cannulas in the umbilicus $(10 \mathrm{~mm})$, subxiphoid $(5 \mathrm{~mm})$, left $(10 \mathrm{~mm})$ and right $(5 \mathrm{~mm})$ lateral subcostal margins. After completion of left liver mobilisation by sectioning the left triangular and coronary ligaments it became evident that the metastasis was too close to the left suprahepatic vein (LSV). This finding was also confirmed by intraoperative U.S.

In an attempt to further mobilise the left liver in order to find a dissection plane for extraparenchymal sectioning of the LSV a conspicuous bleeding was produced by tearing of the vein. Even with lowering of the CVP the bleeding was obstructing the visualization of the venous edges, so impairing the possibility for direct suturing of the vein. To avoid a conversion to laparotomy, the vein was temporarily clamped with a Johann forceps inserted through the right subcostal trocar, then the left lateral sectionectomy was completed to better clear the field from the left liver lobe using the remaining trocars.

Then the vein was released by the Johann forceps compression and was sutured with two endoclips QuickClipPro® (Olympus Medical Systems Corp., Tokyo, Japan). The endoclips were inserted 
through the shaft of a $5 \mathrm{~mm}$ laparoscopic instrument in order to guarantee perfect positioning of the clip on the vein edges (Fig 1). Haemostasis is then strengthened by supplemental interrupted $5 / 0$ prolene sutures. Abdominal drainage was left in place for 24 hours and the patient was discharged on the fourth postoperative day with an uneventful postoperative course. No blood transfusion was required.

\section{Discussion}

The diffusion of laparoscopy as a method to approach liver surgery has been impaired by overall technical challenges, difficulties in obtaining disease-free margin resection and sometimes uncertainty on the most effective hemostatic method. Initially, laparoscopic resection was only suitable for small, peripheral anterior lesions, primarily left-, then also right-sided ${ }^{[5]}$. Major resections were reported by high volume Institutions only ${ }^{[14]}$, whereas the feasibility of wedge resection of deep lesions has been brought by the development of US laparoscopic probes. When facing major hemorrhage, the advantages of open surgery especially in directly identifying the source of bleeding, finger tamponade of the vessel and better exposure for suturing are undeniable ${ }^{[15]}$. The hand is still considered the best "a-traumatic liver retractor by some authors" ${ }^{[16]}$. Therefore, bleeding control remains the pivotal issue when comparing the different approaches. Most of the devices developed for safer and hemostatic liver parenchymal transection are now available for laparoscopic surgery. There are several studies ${ }^{[17-20]}$ that questioned which one of all hemostatic devices should be the best option in terms of decreasing blood loss and post-operation complications but no one seems to overcome to the others ${ }^{[17]}$. Furthermore, there seems no unanimous agreement among the authors. Probably the best option should be left to the surgeon's choice ${ }^{[22]}$. The same principles of inflow and outflow control through an optional use of the Pringle's maneuver and the anesthesiologic lowering of CVP below $5 \mathrm{mmHg}$ apply to laparoscopic surgery, with the only advantage of using a 10 to 14 $\mathrm{mmHg}$ intra-abdominal pressure, always considering the risk of venous embolism ${ }^{[23]}$. Our report addresses the issue of a lesion proximal to a major suprahepatic vein, where the exposure is poor so increasing the need for maximum liver mobilization. Tearing of the vein with minimal residual vessel stump and almost no visibility because of intermittent major bleeding and the need may prompt rapid conversion to open surgery. In this instance, immediate clamping by Johann forceps of the venous proximal stump controlled the bleeding, so allowing for safe completion of resection. With the surgical field cleared by the left liver, we were able to preliminary close the vein with two QuickClipPro® (Olympus Medical Systems Corp., Tokyo, Japan) sequentially displaced on the vein rims. The clip applicator was inserted into the abdomen through the shaft of a $5 \mathrm{~mm}$ laparoscopic instrument for two reasons: 1) avoid loss of $\mathrm{CO} 2$ through the laparoscopic trocar because of the small calibre of the clip applicator; 2) perfect directioning of the pliable Teflon clip applicator.This original technique allows to overcome the limitation of regular laparoscopic clips, which can be used only by lateral approach and require a few millimeters of free stump. The QuickClipPro ${ }^{\circledR}$ as any other endoscopic haemostatic clip are designed to be released by frontal approach and their small tooth grants for durable retention onto the tissue.

\section{Conclusion}

Blood loss still remains a serious complication during laparoscopic liver resections. Intra-operative treatment doesn't seem to occur very often in the international literature. Anyway, an open surgery seems to be the most used therapeutic procedure. However, a careful procedure (in terms of pre-operative imaging and knowledge of the several techniques) should be carried out by experienced operators for every laparoscopic resection in order to avoid even such rare but life-threatening complications.

\section{Conflict of interest}

There was no conflict of interest.

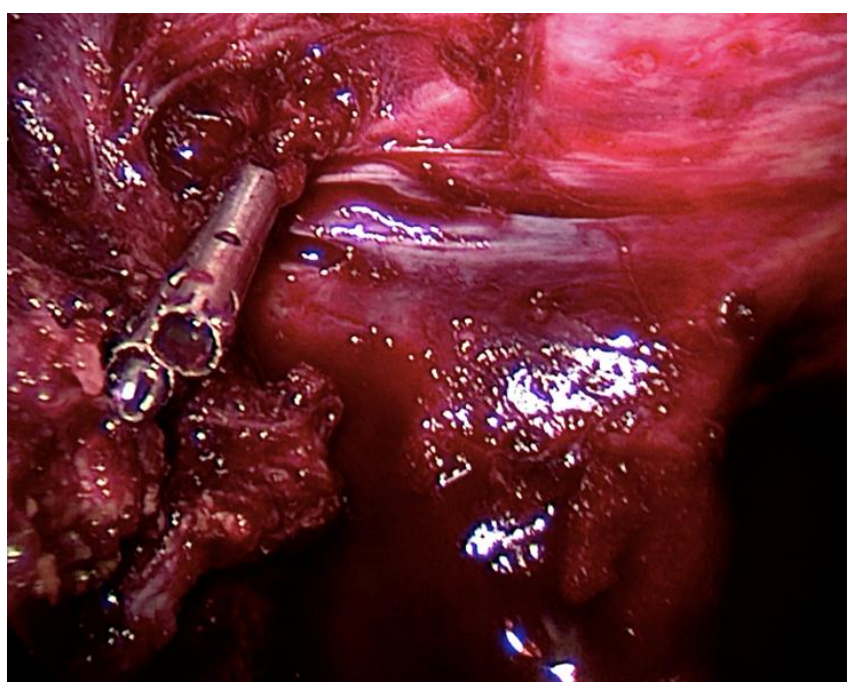

Fig 1: The clip on the vein edges

\section{References}

[1] Giuseppe Di Giuro, Panagiotis Lainas, Dominique Franco, Ibrahim Dagher (2010) Laparoscopic left hepatectomy with prior vascular control Surg Endosc 24:697-699

[2] CheungTT, PoonRT, YuenWK, ChokKS, Jenkins CR, ChanSC, et al. Long-term survival analysis of pure laparoscopic versus open hepatectomy for hepatocellular carcinoma in patients with cirrhosis: a single-center experience. Ann Surg. 2013;257:506-11

[3] LeeKF, CheungYS, ChongCN, TsangYY, NgWW, LingE, etal. Laparoscopic versus open hepatectomy for liver tumours: a case control study. Hong Kong medical journal = Xianggang yi xue za zhi / Hong Kong Academy of Medicine. 2007;13:442-8

[4] Slakey DP, Simms E, Drew B, Yazdi F, Roberts B. Complications of liver resection: Laparoscopic versus open procedures. JSLS. 2013;17:46-55

[5] Mohammad Abu Hilal, Tim Underwood, Matthew G. Taylor, Khaled Hamdan, Hassan Elberm, Neil W. Pearce (2010) Bleeding and hemostasis in laparoscopic liver surgery Surg Endosc 24:572-577

[6] Koji Komeda, MD, PhD, Michihiro Hayashi, MD, PhD, Yoshihiro Inoue, MD, PhD, Tetsunosuke Shimizu, MD, $\mathrm{PhD}$, Mitsuhiro Asakuma, MD, PhD, Fumitoshi Hirokawa, MD, PhD, Yoshiharu Miyamoto, MD, PhD, and Kazuhisa Uchiyama, MD, PhD (2013) TECHNICAL REPORT Clinical Usefulness of Endo Intestinal Clips During Pringle's Maneuver in Laparoscopic Liver Resection: A Technical Report; 23:e103-e105 
[7] Gigot J, Glineur D, Santiago Azagra J, Goergen M, Ceuterick M, Morino M, Etienne J, Marescaux J, Mutter D, van Krunckelsven L, Descottes B, Valleix D, Lachachi F, Bertrand C, Mansvelt B, Hubens G, Saey J, Schockmel R (2002) Hepatobiliary and Pan- creatic Section of the Royal Belgian Society of Surgery and the Belgian Group for Endoscopic Surgery. Laparoscopic liver resection for malignant liver tumors: preliminary results of a multicenter European study. Ann Surg 236:90-97

[8] H. G. Baladas, T. J. Borody, G. S. Smith, M. B. Dempsey, M. A. Richardson, G. L. Falk (2002) Laparoscopic excision of a Brunner's gland hamartoma of the duodenum Surg Endosc $\square$ 2002) 16: 1636 \pm 1639 Ó Springer-Verlag New York Inc. 2002

[9] Schmandra T, Mierdl S, Bauer H, Gutt C, Hanisch E (2002) Transoesophageal echocardiography shows high risk of gas embolism during laparoscopic hepatic resection under carbon dioxide pneumoperitoneum. $\mathrm{Br} \mathrm{J}$ Surg 89:870-876

[10] Jones R, Moulton C, Hardy K (1998) Central venous pressure and its effect on blood loss during liver resection. Br J Surg 85:1058- 1060

[11] Jarnagin WR, Gonen M, Fong Y, DeMatteo RP, BenPorat $\mathrm{L}$, Little $\mathrm{S}$, et al. Improvement in perioperative outcome after hepatic resection: analysis of 1, 803 consecutive cases over the past decade. Ann Surg. 2002;236:397-406

[12] Katz SC, Shia J, Liau KH, Gonen M, Ruo L, Jarnagin WR et al. Operative blood loss independently predicts recurrence and survival after resection of hepatocellular carcinoma. Ann Surg. 2009; 249: 617-23

[13] R.A. Agha, A.J. Fowler, A. Saetta, I. Barai, S. Rajmohan, D.P. Orgill, for the SCARE Group, The SCARE statement: consensus-based surgical case report guidelines, Int. J. Surg. 34 (October) (2016) 180-186.

[14] Alexander Ferko, MD, Ph. D, w Zdene`k S ubrt, MD, Ph. $\mathrm{D},{ }^{*} \mathrm{w}$ Filip $\mathrm{C}^{`} \mathrm{ec}{ }^{`} \mathrm{ka}, \mathrm{MD}, *$ and Bohumil Jon, MD, Ph. D (2010) TECHNICAL REPORT Laparoscopic Right Hepatectomy-Our Approach for Vascular Control: Video Clip ; 20: e10
[15] Tze Yi Low and Brian Kim Poh Goh Emergency handassisted laparoscopic haemostasis for post-operative haemorrhage following laparoscopic liver resection $\mathrm{J}$ Minim Access Surg. 2018 Apr-Jun; 14(2): 171-173.

[16] H. G. Baladas, T. J. Borody, G. S. Smith, M. B. Dempsey, M. A. Richardson, G. L. Falk Laparoscopic excision of a Brunner's gland hamartoma of the duodenum Surg Endosc $\square$ 2002)

[17] Xiao W-K, Chen D, Hu A-B, et al. Radiofrequencyassisted versus clamp-crush liver resection: a systematic review and meta-analysis. J Surg Res [Internet] Elsevier Inc 2014 Apr;187(2):471-83

[18] Curr_o G, Lazzara S, Barbera A, et al. The Aquamantys! system as alternative for parenchymal division and hemostasis in liver resection for hepatocellular carcinoma: a preliminary study 2014;18:2-5.

[19] Pamecha V, Gurusamy KS, Sharma D, Davidson BR. Techniques for liver parenchymal transection: a metaanalysis of randomized controlled trials. HPB (Oxford) [Internet] 2009 Jun;11(4):275-81.

[20] Rahbari NN, Koch M, Schmidt T, et al. Meta-analysis of the clamp- crushing technique for transection of the parenchyma in elective he- patic resection: back to where we started? Ann Surg Oncol [Internet] 2009 Mar;16(3):630-9.

[21] Goro Honda • Masanao Kurata • Yukihiro Okuda • Shin Kobayashi - Sosuke Tadano - Tatsuro Yamaguchi • Hiroshi Matsumoto • Daisuke Nakano • Keiichi Takahashi Totally laparoscopic hepatectomy exposing the major vessels J Hepatobiliary Pancreat Sci (2013) 20:435-440

[22] D.M. Manas, J. Figueras, D. Azoulay, J.C. Garcia Valdecasas, J. French, E. Dixon, N. O'Rourke, N. Grovale, V. Mazzaferro Expert opinion on advanced techniques for hemostasis in liver surgery (2015) EJSO xx (2016) 1e11

[23] Hadrien Tranchart • Nicholas O'Rourke • Ronald Van Dam • Martin Gaillard • Panagiotis Lainas • Atsushi Sugioka • Go Wakabayashi • Ibrahim Dagher Bleeding control during laparoscopic liver resection: a review of literature J Hepatobiliary Pancreat Sci (2015) 